\title{
Relationship of Posttreatment Decentering and Cognitive Reactivity to Relapse in Major Depression
}

\author{
David M. Fresco \\ Kent State University
}

\author{
Zindel V. Segal, Tom Buis, and Sydney Kennedy \\ Centre for Addiction and Mental Health
}

\begin{abstract}
Z. V. Segal et al. (2006) demonstrated that depressed patients treated to remission through either antidepressant medication (ADM) or cognitive-behavioral therapy (CBT), but who evidenced moodlinked increases in dysfunctional thinking, showed elevated rates of relapse over 18 months. The current study sought to evaluate whether treatment response was associated with gains in decentering-the ability to observe one's thoughts and feelings as temporary, objective events in the mind-and whether these gains moderated the relationship between mood-linked cognitive reactivity and relapse of major depression. Findings revealed that CBT responders exhibited significantly greater gains in decentering compared with ADM responders. In addition, high post acute treatment levels of decentering and low cognitive reactivity were associated with the lowest rates of relapse in the 18-month follow-up period.
\end{abstract}

Keywords: major depression, cognitive behavior therapy, decentering, modes of mind

Major depressive disorder (MDD) is a prevalent and debilitating national health problem with lifetime and 12-month prevalence rates of $17 \%$ and $7 \%$, respectively, according to the replication of the National Comorbidity Survey (Kessler, Berglund, et al., 2005; Kessler, Chiu, Demler, Merikangas, \& Walters, 2005). Despite the availability of treatments of established efficacy, there is a growing consensus that the outcome for patients following acute treatment is relatively poor (Fava, Rafanelli, Grandi, Conti, \& Belluardo, 1998). Estimates suggest that approximately $50 \%$ of patients experience a recurrence within 2 years of remission following shortterm treatment (Keller, Lavori, Rice, Coryell, \& Hirschfeld, 1986), and the risk of MDD's becoming a chronic problem increases substantially with each additional episode experienced (Solomon et al., 2000). Because clinical management of patients with depression targets symptom reduction within the episode as the primary goal, little attention has been paid to the development of interventions that provide both symptomatic relief and durable treatment gains.

Hollon, Stewart, and Strunk (2006) reviewed evidence that cognitive-behavioral therapy (CBT) interventions for depression are equivalent to antidepressant medication (ADM) in reducing acute symptoms and are twice as durable, as compared with medication, for reducing rates of relapse or recurrence (Hollon et al., 2006). Findings such as these have generated considerable interest in understanding the mechanisms by which CBT produces these benefits. Hollon et al. (2006) differentiated between two kinds of enduring effects. First, treatment effects refer to factors that mitigate problems that would not have gone away in the absence of some formal intervention. From a research standpoint,

David M. Fresco, Department of Psychology, Kent State University; Zindel V. Segal, Tom Buis, and Sydney Kennedy, Centre for Addiction and Mental Health.

Correspondence concerning this article should be addressed to David M. Fresco, Department of Psychology, Kent State University, Kent, OH 44242. E-mail: fresco@kent.edu interventions can be evaluated for their treatment effects by assessing the problem they putatively target. This assessment is accomplished by demonstrating that the problem does not reoccur or, if it reoccurs, that the frequency and/or intensity is lessened as compared with before the delivery of the intervention. Thus, curative interventions work by eliminating or reversing the underlying processes that would otherwise lead to the continuation of the disorder. Similarly, preventive effects refer to an intervention's capacity to reduce risk for problems in the future-presumably by thwarting the causal processes that could result in the initial onset or recurrence of the disorder. The value of this distinction lies in its suggestion that some treatment-induced changes may be relevant to symptom reduction, whereas others pertain to illness mitigation and future risk. Although the benefits of CBT in producing durable treatment gains for MDD are well established, isolating the mechanisms by which CBT produces these results has proven more elusive. For instance, reductions in negative cognitive products (i.e., the surface-level thoughts or dysfunctional attitudes) do not appear to be the mechanism by which CBT produces its results, as reductions in these thoughts have tended to covary with reductions in depression symptoms, occur in response to noncognitive treatments such as ADM therapy and interpersonal psychotherapy, and show little association with the durability of the treatment gains (cf. Hollon et al., 1992; Hollon \& Kriss, 1984; Imber et al., 1990; Safran, Vallis, Segal, \& Shaw, 1986; Simons, Garfield, \& Murphy, 1984). Rather, changes in cognitive structures, core schema, or core processes are regarded as representing critical change mechanisms in cognitive therapy (Beck, Rush, Shaw, \& Emery, 1979; Safran et al., 1986).

A potentially fruitful avenue for identifying vulnerability factors for depression and mechanisms for durable change resides at the confluence of cognitive science, clinical science, and affective neuroscience (cf. Barnard \& Teasdale, 1991; Brewin \& Power, 1999; LeDoux, 1996; Samoilov \& Goldfried, 2000; Teasdale, 1999). These models all take the view that there exists a complex multilevel relationship between cognitive processing and emo- 
tional processing and distinguish between explicit higher order conceptual processing, which involves primarily rule-based learning, and more rapid, associational processing. Building on this higher and lower processing typology, Teasdale further articulated three modes of mind that have a differential association with emotional disorders such as major depression. Of most relevance to major depression is what Teasdale called mindless emoting, which is associated with purely reactive, sensory-driven emoting without focal awareness of conceptual or schematic meanings. In this mode of mind, individuals' thinking may be characterized as rigid, interlocked processing patterns such as repetitive, ruminative, and negatively self-focused thinking. Another important mode of mind is conceptualizing-doing, which Teasdale characterized as predominated by a focus on conceptual content and analysis (e.g., writing a paper, planning one's day, or solving a logical problem). Finally, Teasdale described mindful experiencing-being, which he characterized as times when individuals are focally aware of thoughts, internal and external sensations, and potential holistic meanings that these multiple sources of information might convey. Of interest, Teasdale's modes-of-mind typology corresponds favorably to the distinctions of emotional mind, reasonable mind, and wise mind, described by Linehan (1993) in dialectical behavior therapy. Further, both theorists have posited that individuals use all three modes throughout their day and that it is commonplace to experience many switches among modes. However, in the context of psychotherapy, helping individuals to cultivate the capacity to process the world from a wise-mind or mindful experiencing-being perspective is regarded as the optimal mode of mind.

Teasdale's (1999) modes-of-mind typology also provides guidance to better understand the cognitive-behavioral model of the etiology and treatment of MDD. For instance, vulnerability for MDD can be regarded as an individual's tendency to slip into a mindless emoting mode when confronted with a stressful event or a potentially transient sad mood. By predominately processing the world through the mindless emoting mode, one becomes more likely to convert a transient sadness into an enduring depression, in part by undermining healthy emotional processing. Similarly, Teasdale cautioned that an implementation of CBT that is overly reliant on cognitive disputation might, in turn, promote an overutilization of a conceptualizing-doing mode of processing to the detriment of acquiring and utilizing mindful experiencing-being. Thus, from this way of thinking, durable CBT for MDD is likely to follow when an individual's predominant mode of mind is characterized by reductions in mindless emoting and increases in mindful experiencing-being.

\section{Cognitive Reactivity}

Cognitive reactivity, defined as a change in one or more cognitive indices in response to an emotion evocation challenge, in the nomenclature of Teasdale (1999), represents a mindless emoting mode of mind as well as a promising marker of psychological vulnerability and increased risk to depression. In the methodology most often used to assess cognitive reactivity, patients are asked to listen to a piece of music designed to make them feel briefly sad, while also recalling a personally upsetting time in their life. In an initial study, Segal, Gemar, and Williams (1999) demonstrated that patients with depression treated to remission with either open-label
ADM or open-label CBT evidenced different patterns of cognitive reactivity following a negative mood induction, and that this cognitive reactivity predicted depression relapse. Specifically, ADM responders demonstrated increases in dysfunctional attitudes, whereas CBT responders demonstrated no change in dysfunctional attitudes. In a replication study using random assignment to treatment condition, Segal et al. (2006) similarly found that ADM responders evidenced significantly more cognitive reactivity as compared with CBT responders $(d=0.42)$. Further, treatment responders, irrespective of whether they received ADM or CBT, who also evidenced cognitive reactivity (equivalent to an 8-or-more-point increase in dysfunctional attitudes following an emotion evocation challenge), were at increased risk of relapse in the subsequent 18 months as compared with participants with minimal or no cognitive reactivity. Findings from these two studies provide evidence that cognitive reactivity following an emotion evocation challenge may confer vulnerability for depression relapse or recurrence and that CBT may target this vulnerability more effectively than ADM.

\section{Decentering}

The current study represents a secondary analysis of Segal et al. (2006) with the aim of identifying treatment-related factors that might moderate the association of cognitive reactivity and relapse in this group of patients. Decentering is described as the capacity to take a present-focused, nonjudgmental stance in regard to thoughts and feelings and to accept them. The construct of decentering is of particular interest, as recent conceptualizations of cognitive treatment suggest that decentering may reduce levels of depressive rumination by teaching patients more adaptive ways of relating to their thinking (Segal, Williams, \& Teasdale, 2002). However, decentering has also been a topic of discussion early on in the cognitive-behavioral movement. Both Beck et al. (1979) and Safran and Segal (1990) have described it as an important ingredient of change in cognitive therapy. Ingram and Hollon (1986) posited not only that decentering was important in helping to reduce current depression symptoms but also that "the long term effectiveness of cognitive therapy may lie in teaching patients to initiate this process in the face of future stress" (p. 272). Decentering also corresponds to the mindful experiencing-being mode of mind within Teasdale's (1999) typology.

Initial efforts to empirically evaluate the relationship of decentering to depression and to the treatment of depression arose through the study of metacognitive awareness, a construct related to decentering. Metacognitive awareness refers to "the process of experiencing negative thoughts and feelings within a decentered perspective" (Teasdale et al., 2002, p. 276) and is typically assessed with the Measure of Awareness and Coping in Autobiographical Memory (MACAM; Moore, Hayhurst, \& Teasdale, 1996), a semistructured clinical interview. In this study, 158 patients who had achieved partial remission of MDD following treatment with $\mathrm{ADM}$ were randomized to receive $\mathrm{ADM}$ and clinical management alone or together with cognitive therapy for 20 weeks and then receive follow-up continuation and maintenance medication for 48 weeks. Findings indicated that lower levels of baseline metacognitive awareness predicted earlier relapse across both treatment groups. There was also a larger increase in meta- 
cognitive awareness in the cognitive therapy group as compared with the clinical management group.

\section{Measurement of Decentering}

Although the findings associated with metacognitive awareness are promising, the MACAM is time intensive and requires clinician assessment. Consequently, Fresco and colleagues (in press) undertook a psychometric analysis of the Experiences Questionnaire (EQ), a rationally derived, self-report measure designed by Teasdale to measure both decentering (and related processes) and rumination in the context of mood disorders. The EQ was designed as a relatively brief, practical measure of the primary psychotherapy process in mindfulness based cognitive therapy (MBCT) but had not undergone rigorous psychometric evaluation. Using exploratory and confirmatory factor analysis in two consecutive large samples of college students, an 11-item decentering factor emerged. This factor structure was again confirmed in a sample of patients with remitted MDD. Findings from this latter sample revealed that healthy control participants endorsed higher levels of decentering as compared with MDD patients $(d=1.3)$ and that decentering was significantly and negatively correlated with levels of clinician-assessed and self-reported symptoms of depression in the patients with MDD.

\section{The Present Study}

The present study represents a secondary analysis of Segal et al. (2006), who found that cognitive reactivity (a form of mindless emoting mode of mind) in conjunction with an emotion evocation challenge predicted relapse in the subsequent 18-month span. We sought to evaluate three additional hypotheses. First, we predicted that patients who achieved a positive treatment response following random assignment to CBT would evidence significantly greater gains in decentering (a mindful experiencing-being mode of mind) as compared with patients with a positive treatment response to ADM. Second, we predicted that CBT and ADM responders would show equivalent reductions in dysfunctional attitudes (an index of cognitive content). Third, low cognitive reactivity has already been shown to predict a more durable treatment response (Segal et al., 2006). Thus, we predicted that posttreatment levels of decentering would also show a positive association with treatment durability, particularly in conjunction with low levels of cognitive reactivity.

\section{Method}

\section{Participants}

Participants for the current study were 111 patients with MDD who accepted random assignment to either ADM $(n=43)$ or CBT $(n=68)$ and completed the treatment as well as study measures. Segal et al. (2006) screened 484 patients and, after applying exclusion criteria, randomly assigned 301 patients to either ADM $(n=149)$ or CBT $(n=152)$. Patients not invited for the acute treatment phase of the study were disqualified for not meeting diagnostic criteria for MDD; endorsing the presence of another psychiatric condition judged to require more immediate treatment; having a comorbid diagnosis of borderline, antisocial, or schizotypal personality disorder; having evidence of a prior poor re- sponse to study interventions; and having scheduling difficulties. One hundred forty-four patients who enrolled in the acute treatment phase completed treatment. The attrition following initial consent for randomization occurred prior to the onset of treatment ( $n=69)$, within the first four sessions $(n=51)$, or at some later point in the course of acute treatment $(n=37)$. Refusal of randomization or disappointment with treatment assignment, particularly to ADM, was the most frequent explanation given for study attrition.

Patients accepting initial randomization were approximately 37 years old $(S D=11.5)$; were primarily female $(62 \%)$; and had, on average, experienced $1.37(S D=0.48)$ lifetime episodes of major depression, with the current episode having lasted approximately 33 weeks $(S D=24)$. As reported by Segal et al. (2006), demographic characteristics were unrelated to patient attrition or treatment response. CBT patients received 20 individual weekly sessions over a 22- to 24-week span. ADM patients received 10 to 13 individual sessions with a study psychiatrist over a 26-week span. Assessment of treatment response and posttreatment levels of decentering were completed on the day of the last treatment session. For treatment responders, the mood priming challenge procedure occurred between 3 and 7 days following the posttreatment assessment.

\section{Measures}

Self-report measures. The Decentering subscale of the EQ (Fresco et al., in press) is an 11-item self-report instrument that assesses the construct of decentering. Sample items include "I am better able to accept myself as I am" and "I can observe unpleasant feelings without being drawn into them." In the current study, the Decentering subscale evidenced good internal consistency at both pretreatment $(\alpha=.81)$ and posttreatment $(\alpha=.84)$.

The Dysfunctional Attitude Scale (DAS; Weissman \& Beck, 1978 ) is a 40-item self-report inventory used to assess dysfunctional thinking. Participants rate the degree to which they agree or disagree with statements that assess dysfunctional thoughts on a 7-point Likert-type scale with response options ranging from totally agree to totally disagree. The DAS has previously shown strong internal consistency ( $\alpha=.89$; Weissman, 1979). In the present study, the comparable 40-item Versions A and B were used. Participants received a version of the DAS at the outset of the study, following the completion of treatment as well as just before and immediately following an emotion evocation challenge.

The Beck Depression Inventory-II (BDI-II; Beck, Steer, \& Brown, 1996) is a 21-item self-report measure that assesses the affective, cognitive, behavioral, and somatic symptoms of depression as well as motivational components and suicidal wishes. Items reflect a 2-week time period and are rated on a 4-point scale. In the current study, the BDI-II was used to assess for the recurrence of major depression post acute treatment.

Clinician-assessed measures. The Structured Clinical Interview for $D S M-I V$, Research Version (SCID; First, Spitzer, Gibbon, \& Williams, 1996) is a widely used semistructured interview to determine current and lifetime diagnoses of Axis I disorders. Ventura, Liberman, Green, Shaner, and Mintz (1998) reported high interrater agreement for current diagnosis with an overall weighted kappa of .82. Kappas for MDD are good (.80) to excellent (.91). 
The Hamilton Rating Scale for Depression (HRSD; Hamilton, 1960) is a clinician-administered measure of depression symptom severity that correlates highly with the Beck Depression Inventory (BDI; Beck et al., 1979). The present study used the 17-item version of the HRSD. Acute treatment response was assessed with the HRSD using a criterion of scoring less than 8 (cf. Frank et al., 1991; Jacobson et al., 1996; Zimmerman, Chelminski, \& Posternak, 2004). Using this criterion, 79\% of AMD patients (34/43) and $68 \%$ of CBT patients $(46 / 68)$ were classified as responders.

The Longitudinal Interview Follow-up Evaluation (LIFE; Keller et al., 1987) is a semistructured interview used to measure participants' recovery from or occurrence of new depressive episodes and the occurrence of relapse in depression. In the current study, the LIFE interview in conjunction with phone and mail contact with participants was used to determine relapse of MDD. Patients who endorsed elevations in depression were brought in and evaluated by a clinician with the HRSD. Any patient scoring 15 or greater on the BDI-II or 16 or greater on the HRSD-17 was reinterviewed in a week's time. If their scores remained in the same range, they were assessed with the LIFE to determine whether their level of symptomatology met criteria for MDD. Patients were judged to have relapsed if they were given a diagnosis of MDD at any time during the follow-up.

\section{Treatment Procedures}

Medication treatment. Patients in the ADM condition were treated with one of three first-line, antidepressant medications (sertraline, 50-200 mg; paroxetine, 20-50 mg; or venlafaxine, $75-225 \mathrm{mg}$ ) for a period of 6 months. Choice of medication was naturalistic and based on the treating psychiatrist's clinical judgment. Patients who failed to achieve an adequate response to the initial ADM were allowed to discontinue it and start on a second medication. Patients who failed both trials were removed from the study and offered alternative care. Pharmacotherapy sessions were $20 \mathrm{~min}$ in duration and followed the recommendations for clinical management developed by Fawcett, Epstein, Fiester, Elkin, and Autry (1987).

Cognitive therapy. Patients in the CBT condition received a course of 20 individual weekly sessions over a 22- to 24-week span according to the manualized protocol developed by Beck et al. (1979). Treatment fidelity was assessed using the Cognitive Therapy Scale (Vallis, Shaw, \& Dobson, 1986) by applying ratings of 18 audiotapes (early, middle, and late sessions) from 6 randomly chosen patients. The mean rating for each of the two raters across all tapes was 46.94 and 47.35 , and the level of interrater agreement (intraclass correlation coefficient) was .83 .

\section{Emotion Evocation Challenge}

Patients listened through headphones to a piece of music presented on a CD player while following instructions to recall a time in their lives when they felt sad. The piece of music was "Russia Under the Mongolian Yoke," composed by Sergei Prokofiev (1934/1987). This piece was remastered at half speed and runs for approximately $8 \mathrm{~min}$. This piece, played at half speed, has been shown to be very effective in inducing a negative or depressed mood (e.g., Fresco, Heimberg, Abramowitz, \& Bertram, 2006; Segal et al., 1999, 2006). A meta-analysis of musical mood induction procedures found a good effect size (Cohen's $f=.56$ ) for sad mood inductions including, but not limited to, this Prokofiev piece played at half speed (Westermann, Spies, Stahl, \& Hesse, 1996).

\section{Results}

\section{Baseline Measures}

The first set of analyses examined patients randomly assigned to $\mathrm{ADM}$ or $\mathrm{CBT}$ on baseline measures of depression symptoms, decentering, and dysfunctional attitudes. Findings indicated that the two treatment groups did not differ significantly on baseline measures of self-reported depression, $F(1,110)=0.29, n s$; clinician-assessed depression, $F(1,110)=0.39, n s$; decentering, $F(1,110)=0.03, n s ;$ or dysfunctional attitudes, $F(1,110)=1.00$, $n s$. Sample means and standard deviations are presented in Table 1.

\section{Cognitive Change as a Function of Treatment Response}

The next set of analyses evaluated the degree of change on cognitive measures as a function of treatment condition and re-

Table 1

Means and Standard Deviations for Depression Symptom and Cognitive Measures as Assessed Among Treatment Responders at Pretreatment and Post Acute Treatment

\begin{tabular}{|c|c|c|c|c|c|c|c|c|c|c|c|c|}
\hline \multirow[b]{3}{*}{ Measure } & \multirow{2}{*}{\multicolumn{2}{|c|}{$\begin{array}{l}\text { CBT patients } \\
(n=68): \\
\text { Pre }\end{array}$}} & \multirow{2}{*}{\multicolumn{2}{|c|}{$\begin{array}{l}\text { ADM patients } \\
(n=43): \\
\text { Pre }\end{array}$}} & \multicolumn{4}{|c|}{$\begin{array}{c}\text { CBT responders } \\
(n=46)\end{array}$} & \multicolumn{4}{|c|}{$\begin{array}{l}\text { ADM responders } \\
\quad(n=34)\end{array}$} \\
\hline & & & & & \multicolumn{2}{|c|}{ Pre } & \multicolumn{2}{|c|}{ Post } & \multicolumn{2}{|c|}{ Pre } & \multicolumn{2}{|c|}{ Post } \\
\hline & $M$ & $S D$ & $M$ & $S D$ & $M$ & $S D$ & $M$ & $S D$ & $M$ & $S D$ & $M$ & $S D$ \\
\hline \multicolumn{13}{|l|}{ Depression symptoms } \\
\hline BDI-II & 31.66 & 9.24 & 32.21 & 10.26 & 31.19 & 8.89 & 6.68 & 6.25 & 30.05 & 7.55 & 7.62 & 5.18 \\
\hline HRSD & 19.10 & 3.50 & 18.24 & 4.45 & 19.13 & 3.52 & 4.20 & 1.82 & 18.50 & 4.92 & 3.91 & 2.08 \\
\hline \multicolumn{13}{|l|}{ Cognitive measures } \\
\hline Decentering & 1.65 & 0.59 & 1.74 & 0.52 & 1.76 & 0.64 & 2.42 & 0.42 & 1.70 & 0.52 & 2.15 & 0.50 \\
\hline Dysfunctional attitudes & 164.03 & 32.13 & 152.75 & 36.41 & 157.00 & 31.90 & 126.07 & 28.94 & 161.24 & 27.85 & 132.14 & 27.63 \\
\hline
\end{tabular}

Note. $\quad \mathrm{CBT}=$ cognitive-behavioral therapy; $\mathrm{ADM}=$ antidepressant medication; Pre $=$ pretreatment; Post $=$ post acute treatment; $\mathrm{BDI}-\mathrm{II}=\mathrm{Beck}$ Depression Inventory_II; HRSD = Hamilton Rating Scale for Depression; Decentering = Experiences Questionnaire—Decentering subscale. 
sponse status. A series of 2 (response status: responder, nonresponder) $\times 2$ (time: pretreatment, post acute treatment) repeated measures analyses of variance with decentering and dysfunctional attitudes as the dependent measures were conducted separately for $\mathrm{ADM}$ and $\mathrm{CBT}$ patients. The findings were evaluated using customary standards of significance (e.g., $p<.05$ ) as well as Cohen's (1988) effect size conventions for $f$ (small $=.10$, medium $=.25$, and large $=$ $.40)$ and $d$ (small $=0.20$, medium $=0.50$, and large $=0.80$ ).

Decentering. Our first hypothesis posited that CBT responders would evidence significantly greater gains in decentering as compared with ADM responders. Findings for ADM patients revealed a significant main effect for time, $F(1,42)=11.74, p=.002, f=.56$; a nonsignificant main effect for response status, $F(1,42)=1.22$, $n s$, $f=.18$; and a nonsignificant Response Status $\times$ Time interaction, $F(1,42)=1.58, n s, f=.21$. CBT patients evidenced a significant main effect for time, $F(1,67)=51.47, p<.001, f=.85$, and a nonsignificant main effect for response status, $F(1,67)=3.05, p=$ $.08, f=.21$. However, these main effects were qualified by a significant Response Status $\times$ Time interaction, $F(1,67)=8.05, p=.006$, $f=.34$. Examination of the group means presented in Table 1, coupled with this interaction effect, revealed that CBT treatment responders evidenced significantly larger gains in decentering as compared with CBT nonresponders.

Dysfunctional attitudes. Our second hypothesis posited that CBT responders and ADM responders would evidence equivalent drops in dysfunctional attitudes. Findings for ADM patients revealed a significant main effect for time, $F(1,42)=14.97, p<$ $.001, f=.60$; a nonsignificant main effect for response status, $F(1$, $42)=2.19, n s, f=.22$; and a nonsignificant Response Status $\times$ Time interaction, $F(1,42)=0.73, n s, f=.14$. Similarly, CBT patients evidenced a significant main effect for time, $F(1,67)=$ 26.68, $p<.001, f=.64$; a nonsignificant main effect for response status, $F(1,67)=0.56, n s, f=.10$; and a nonsignificant Response Status $\times$ Time interaction, $F(1,67)=1.86, n s, f=.17$. Examination of the group means presented in Table 1, coupled with this nonsignificant interaction effect, indicated that ADM and CBT patients experienced drops in dysfunctional attitudes over the course of acute treatment that occurred irrespective of treatment response status.

\section{Cognitive Change Among Treatment Responders}

Univariate analyses of covariance were conducted among treatment responders to assess whether posttreatment levels of decentering or dysfunctional attitudes differed as a function of treatment condition while controlling for the corresponding baseline measure of either decentering or dysfunctional attitudes. For decentering, findings revealed a significant main effect for treatment condition, $F(1,77)=$ $6.45, p=.013, d=0.58$, indicating that CBT responders evidenced significantly larger gains in decentering as compared with ADM responders when we controlled for baseline levels of decentering. By contrast, CBT responders and ADM responders did not differ significantly from one another on posttreatment levels of dysfunctional attitudes, $F(1,77)=1.49, n s, d=0.28$.

\section{Association of Cognitive Reactivity and Decentering With Relapse}

Segal et al. (2006) reported that 99 patients who completed and responded to either $\mathrm{ADM}$ or $\mathrm{CBT}$ entered the emotion evocation and 18-month follow-up phase of the study. The findings indicated that the emotion evocation challenge successfully induced shortlived sadness and that some patients who had recovered from depression evidenced cognitive reactivity that, in turn, predicted likelihood of relapse (see Segal et al., 2006, for details).

Another aim of the current study was to examine the relationship of posttreatment decentering to cognitive reactivity and relapse. Specifically, our third hypothesis predicted that a combination of high posttreatment decentering and low cognitive reactivity would be associated with the most durable treatment response during the follow-up period. This prediction lends itself to a moderation analysis, where the interaction of a known predictor of relapse (cognitive reactivity) is examined in relation to a putative moderator of that main effect relationship. Kraemer, Stice, Kazdin, Offord, and Kupfer (2001) recently provided a cogent statement that offers clarity on methodological and statistical issues in evaluating both moderators and mediators. A thorough review of this report is beyond the scope of the current study, but given the relative statistical independence between decentering and cognitive reactivity $(r=-.11)$ and the design of data acquisition, the following analysis qualifies by Kraemer et al.'s standards as an appropriate test of moderation. ${ }^{1}$ To accomplish this aim, a Cox proportional-hazards survival analysis was computed to estimate the impact of posttreatment decentering and cognitive reactivity on time until relapse among acute treatment responders. Treatment condition (ADM or CBT), posttreatment depression symptoms (to control for posttreatment depression levels), posttreatment decentering, and cognitive reactivity were entered at the first step as main effects. The nested two-way interactions composed of treatment condition, posttreatment decentering, and cognitive reactivity were entered at Step 2, followed by the three-way interaction of these variables. The overall model resulted in a significant drop in the survival function value, $\chi^{2}(8, N=77)=21.28, p=.006$. In the full model, cognitive reactivity $(B=-0.66, S E=0.22)$, Wald $(1)=8.55, p=.003$, and the three-way interaction of treatment condition, posttreatment decentering, and cognitive reactivity were significant $(B=0.24, S E=0.10)$, Wald $(1)=4.86$, $p=.028$. This interaction was interpreted by solving the Cox regression model for treatment condition $(\mathrm{ADM}=0 ; \mathrm{CBT}=1)$ at either 1 standard deviation above or 1 standard deviation below the sample mean on posttreatment decentering as well as the definition of a marked increase (DAS change $\geq 8$ ) versus minimal or no cognitive reactivity (DAS change $<8$ ). Solving the regression equation in this manner estimated the proportional hazards for each treatment condition as a function of low decentering-high cognitive reactivity, low decentering-minimal cognitive reactivity, high decentering-high cognitive reactivity, and high decenteringminimal cognitive reactivity. As seen in Figures 1 and 2, findings revealed that a combination of high decentering and low cognitive reactivity was associated with the lowest predicted rates of relapse

\footnotetext{
${ }^{1}$ As pointed out by an anonymous reviewer, there may be theoretically interesting mediation relationships between decentering and cognitive reactivity. However, by Kraemer et al.'s (2001) standards, the methodology of this secondary analysis is disqualified by virtue of the fact that the putative mediator (decentering) was not assessed prior to the independent variable (cognitive reactivity) and the two variables are not significantly correlated.
} 


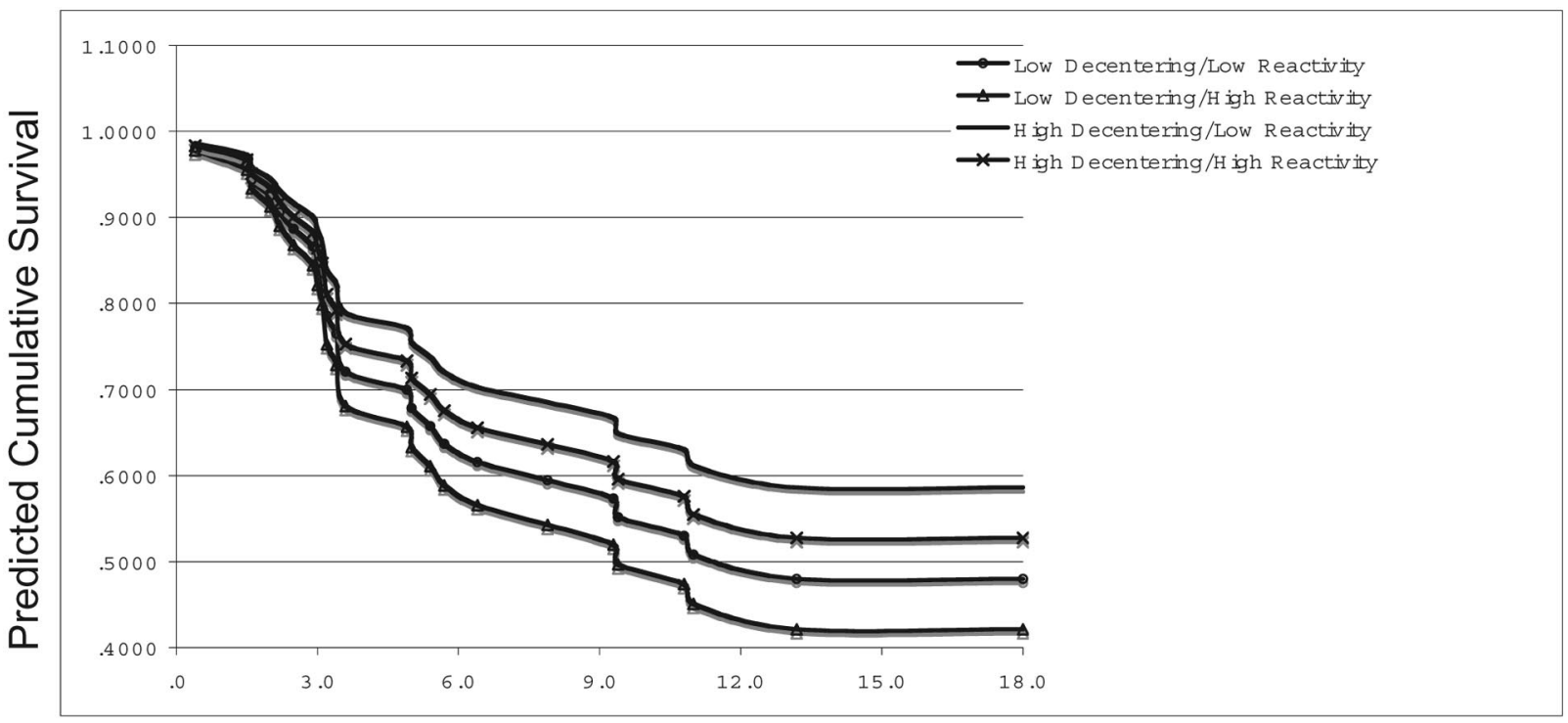

\section{8-Month Follow-up Period}

Figure 1. Survival analysis showing the predict rates of survival among antidepressant medication responders as a function of high and low decentering and high and low cognitive reactivity.

(e.g., highest rates of survival) particularly among CBT responders. By contrast, and unexpectedly, among CBT responders with high decentering and high cognitive reactivity, the predicted survival rate was markedly diminished. Similarly, among CBT responders with low decentering, the relationship between cognitive reactivity and relapse evidenced an opposite relationship such that the combination of low decentering and high cognitive reactivity was associated with a more durable treatment response as compared with low decentering and low cognitive reactivity.

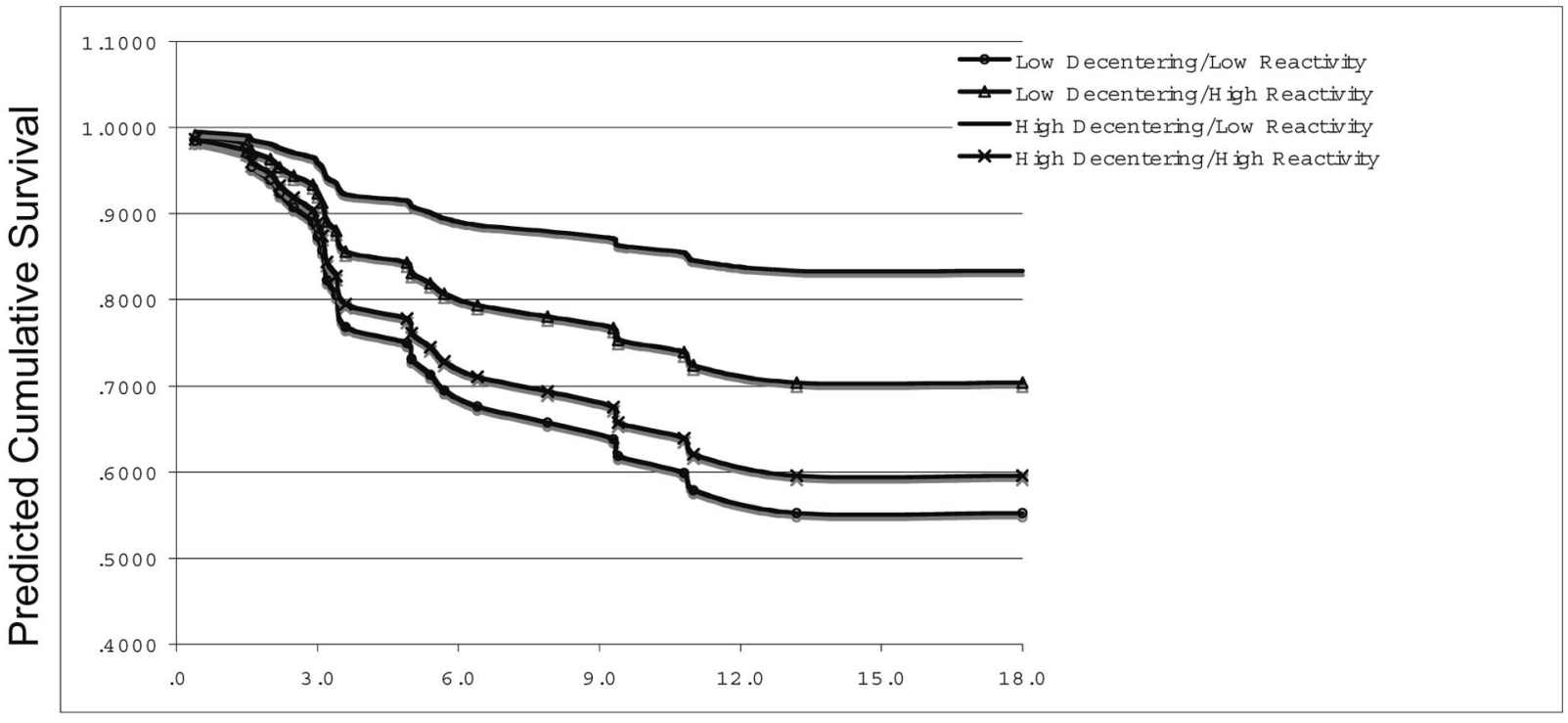

18-Month Follow-up Period

Figure 2. Survival analysis showing the predict rates of survival among cognitive-behavioral therapy responders as a function of high and low decentering and high and low cognitive reactivity. 
In an attempt to better understand the relationship of decentering to relapse, one additional survival analysis was conducted, this time examining the relationship of high decentering and low decentering at the sample average of cognitive reactivity. Among CBT responders, the predicted cumulative survival associated with high decentering was .73, whereas the predicted cumulative survival for CBT responders with low decentering was .66. For ADM responders, the predicted cumulative survival associated with high decentering was .55 , whereas the predicted cumulative survival associated with low decentering was .46 .

\section{Discussion}

The main focus of the current study was to investigate the relationship of decentering, which is defined as the ability to observe one's thoughts and feelings as temporary, objective events in the mind, to both recovery from major depression and protection against relapse. We can now report two main findings with respect to decentering: First, gains in decentering are more pronounced among CBT treatment responders as compared with ADM responders. Second, high posttreatment decentering appears to offer some protection against relapse of MDD, particularly for patients who recovered in CBT.

We examined the relationship of treatment modality (ADM or CBT) and response status to two self-report cognitive measures: the DAS, a measure of dysfunctional attitudes, and the EQ, a measure of decentering. Similar to other studies (Hollon et al., 1992; Simons et al., 1984), findings indicated that patients, irrespective of treatment condition and response status, evidenced drops in dysfunctional attitudes. However, treatment responders in the CBT condition endorsed significantly larger gains in decentering compared with CBT nonresponders, with this finding corresponding to a medium effect. Similarly, CBT responders evidenced significantly larger gains in decentering compared with $\mathrm{ADM}$ responders, a finding that also corresponded to a medium effect. Thus, these findings indicate that gains in decentering demonstrate some specificity to treatment modality.

This first set of findings from the current study also corresponds favorably to findings reported by Teasdale et al. (2002), despite some important methodological differences. In the Teasdale et al. (2002) study, patients with depression who had achieved a partial remission of their depression with $\mathrm{ADM}$ were randomly assigned to 20 weeks of continued medication management (CM) or 20 weeks of CM plus 16 sessions of CBT. Metacognitive awareness, the process of experiencing negative thoughts and feelings within a decentered perspective (as assessed with the MACAM), increased significantly for patients who received the CBT augmentation of CM. The gains in metacognitive awareness enjoyed by the CM-enriched-with-CBT group held even after controlling for relapse status in the follow-up period and in concurrent levels of depression. Thus, in both studies, gains in metacognitive awareness or decentering appear to be a by-product of receiving CBT. The main methodological difference between the current study and the Teasdale et al. (2002) study was that the current study enrolled participants who met criteria for MDD and randomly assigned them to either $\mathrm{ADM}$ or $\mathrm{CBT}$ to treat their depression. By contrast, the Teasdale et al. (2002) sample consisted of patients with partially remitted depression who were randomly assigned to ADM with $\mathrm{CM}$ with and without CBT. Still, in both studies, gains in either metacognitive awareness or decentering occurred after receiving CBT.
The second focus of the current study was to examine the relationship of decentering to the durability of treatment gains following acute treatment response with either ADM or CBT. Segal et al. (2006), from which the current study emerged, reported that participants in CBT, compared with ADM, evidenced less cognitive reactivity in response to a laboratory emotion evocation challenge, and the cognitive reactivity, irrespective of treatment modality, was associated with earlier relapse in the subsequent 18 months. Findings from the current study complement the earlier results of Segal et al. (2006) by showing that posttreatment levels of decentering in combination with low cognitive reactivity were associated with the most durable treatment response, particularly among CBT responders. The interaction of posttreatment decentering, treatment modality, and cognitive reactivity significantly improved prediction of relapse after controlling for posttreatment levels of depressive symptoms. Overall, the results indicate that high decentering as assessed with the EQ provides protection against relapse in conjunction with low cognitive reactivity. Future researchers may wish to further examine the additive and interactive relationships between decentering and cognitive reactivity to further elucidate their contribution to risk for relapse. The other unexpected finding was associated with CBT responders with low posttreatment decentering who seemed to have a more durable treatment response in association with high cognitive reactivity. One possible explanation for this finding is that low decentering may assess a general inattention to one's thoughts and feelings and that it thus represents a proxy for distraction or perhaps an attentional control strategy that draws the mind away from self-focused attention (cf. Wells, 1990; Wells \& Matthews, 1994). Similarly, the finding of a fragile recovery associated with low decentering and low cognitive reactivity among CBT patients seems compatible with evolutionary accounts of depression such as the emotion context insensitivity hypothesis (Rottenberg, Gross, \& Gotlib, 2005), which posits that depression represents a general psychological inertia characterized by a lack of emotional reactivity for both negative and positive emotional situations. In the short run, this reduced reactivity spares the organism from emotional upheaval and actions that might be counterproductive, but in the long run, this inactivity comes with costs, including greater depression severity, longer episodes of depression, and lower levels of psychosocial functioning (Rottenberg, Kasch, Gross, \& Gotlib, 2002). Perhaps the poor durability associated with low decentering and low cognitive reactivity is a proxy for the lack of emotional reactivity proposed by Rottenberg and colleagues to promote longer and more severe depressive episodes. This question remains an important area of future research.

Teasdale et al. (2002) found that high levels of metacognitive awareness, assessed prior to a patient's enrollment in CM or CM enriched with CBT, improved the prediction of relapse even after controlling for concurrent levels of depressive symptoms. The effects of ADM on metacognitive awareness were not reported. By contrast, in the current study, CBT but not ADM resulted in gains in decentering, and the relationship of decentering to relapse was most pronounced in CBT patients, particularly in conjunction with low cognitive reactivity.

Using the rubric for investigating the enduring effects of treatments proposed by Hollon et al. (2006), these findings offer initial evidence that gains in decentering represent a property of $\mathrm{CBT}$ that is more than simply palliative. Both $\mathrm{ADM}$ and $\mathrm{CBT}$ were equivalent in their 
success in treating the symptoms of the current major depressive episode, and similar numbers of patients in each condition enjoyed a response such that they no longer met criteria for MDD following the end of acute treatment. However, decentering contributed to the durability of CBT, particularly for patients who also evidenced low cognitive reactivity. Although these findings are necessarily tentative, they offer evidence that decentering corresponds to the Hollon et al. (2006) designation of a curative agent within CBT.

\section{Limitations}

Findings from the current study, although encouraging, must be interpreted in the context of some important limitations. First, the current study represents a secondary analysis of another study. The hypotheses proposed in the current study were not central to the earlier investigation, and thus, methodological decisions made in the original study, such as the schedule for the administration of measures such as decentering, prevent us from drawing any conclusions of a causal nature regarding the role of decentering in the process of recovery from MDD. Rather, the findings show that gains in decentering covary with recovery from MDD. Future research is needed that assesses decentering during the course of treatment and prior to the determination of acute treatment response before statements about its role as a mechanism of change can be evaluated (cf. DeRubeis \& Feeley, 1990; Hollon, DeRubeis, \& Evans, 1987; Jacobson et al., 1996). Findings of the current study represent just the second statement to attest to the psychometric properties and predictive validity of the Decentering scale of the EQ (Fresco et al., in press). Although the present results are encouraging, the accumulation of more research evaluating the performance and assessment of decentering of the EQ is needed.

\section{Future Directions}

Three additional questions and areas of future research arise from the findings of the current study. First, the Decentering scale of the EQ represents a new candidate measure assessing a metacognitive factor and mindful experiencing-being mode of mind associated with recovery and relapse prevention. However, a fruitful area of future research will be to evaluate how the EQ relates to other measures of metacognitive abilities. One measure, already discussed, is the MACAM (Moore et al., 1996; Teasdale et al., 2002). Another promising measure of metacognitive ability is the frequency with which individuals endorse extreme response categories, positive or negative, on self-report measures of cognition, such as the Attributional Style Questionnaire (Peterson et al., 1982) and the DAS (Weissman \& Beck, 1978). Teasdale and colleagues (2001) found that higher rates of extreme responding predicted relapse and differential response to cognitive therapy for depression. Similarly, Petersen et al. (2007) found that reductions in extreme responding predicted full depression remission over 8 weeks of treatment with ADM. Furthermore, during the maintenance phase, patients randomly assigned to ADM-only maintenance evidenced significantly greater increases in extreme responding compared with patients in ADM maintenance augmented with CBT. Second, Teasdale's (1999) modes-ofmind model posits that patterns of cognitive rigidity such as cognitive reactivity and depressive rumination confer risk for occurrence-recurrence of MDD. An important follow-up to the current study will be to simultaneously evaluate the relationship of cognitive reactivity, depressive rumination, and decentering in the context of recovery from MDD and in the emotion evocation challenge methodology. A particular question that remains is whether gains in decentering simply reflect drops in depressive rumination or whether gains in decentering represent the acquisition of a metacognitive ability to counteract its effects. Third, findings from the current study show that a traditional implementation of CBT for depression (Beck et al., 1979) produced gains in decentering. The version of CBT used in the current study did not include any of the modifications and additions found in MBCT (Segal, Williams, \& Teasdale, 2002), even though one of the current investigators contributed to the MBCT protocol. A fruitful area of future research will be to see whether MBCT protects against MDD relapse via gains in a mindful experiencing-being mode of mind while decreasing a mindless emoting mode of mind.

\section{References}

Barnard, P. J., \& Teasdale, J. D. (1991). Interacting cognitive subsystems: A systemic approach to cognitive-affective interaction and change. Cognition and Emotion, 5, 1-39.

Beck, A. T., Rush, A. J., Shaw, B. F., \& Emery, G. (1979). Cognitive therapy of depression. New York: Guilford Press.

Beck, A. T., Steer, R. A., \& Brown, G. K. (1996). Manual for the BDI-II. San Antonio, TX: Psychological Corporation.

Brewin, C. R., \& Power, M. (1999). Integrating psychological therapies: Processes of meaning transformation. British Journal of Medical Psychology, 72, 143-157.

Cohen, J. (1988). Statistical power analysis for the behavioral sciences (2nd ed.). San Diego, CA: Academic Press.

DeRubeis, R. J., \& Feeley, M. (1990). Determinants of change in cognitive therapy for depression. Cognitive Therapy and Research, 14, 469-482.

Fava, G. A., Rafanelli, C., Grandi, S., Conti, S., \& Belluardo, P. (1998). Prevention of recurrent depression with cognitive behavioral therapy: Preliminary findings. Archives of General Psychiatry, 55, 816-820.

Fawcett, J., Epstein, P., Fiester, S. J., Elkin, I., \& Autry, J. H. (1987). Clinical management: Imipramine/placebo administration manual: NIMH Treatment of Depression Collaborative Research Program. Psychopharmacology Bulletin, 23, 309-324.

First, M. B., Spitzer, R. L., Gibbon, M., \& Williams, J. B. W. (1996). Structured clinical interview for DSM-IV Axis I disorders-Patient edition (SCID-I/P, Version 2.0). New York: New York State Psychiatric Institute, Biometrics Research Department.

Frank, E., Prien, R. F., Jarrett, R. B., Keller, M. B., Kupfer, D. J., Lavori, P. W., Rush, A. J., \& Weissman, M. M. (1991). Conceptualization and rationale for consensus definitions of terms in major depressive disorder. Archives of General Psychiatry, 48, 851-855.

Fresco, D. M., Heimberg, R. G., Abramowitz, A., \& Bertram, T. L. (2006). The effect of a negative mood priming challenge on dysfunctional attitudes, explanatory style, and explanatory flexibility. British Journal of Clinical Psychology, 45, 167-183.

Fresco, D. M., Moore, M. T., van Dulmen, M., Segal, Z. V., Teasdale, J. D., Ma, H., \& Williams, J. M. G. (in press). Initial psychometric properties of the Experiences Questionnaire: A self-report survey of decentering. Behavior Therapy.

Hamilton, M. (1960). A rating scale for depression. Journal of Neurology, Neurosurgery, and Psychiatry, 23, 56-62.

Hollon, S. D., DeRubeis, R. J., \& Evans, M. D. (1987). Causal mediation of change in treatment for depression: Discriminating between nonspecificity and noncausality. Psychological Bulletin, 102, 139-149.

Hollon, S. D., DeRubeis, R. J., Evans, M. D., Wiemer, M. J., Garvey, M. J., 
Grove, W. M., \& Tuason, V. B. (1992). Cognitive therapy and pharmacotherapy for depression: Singly and in combination. Archives of General Psychiatry, 49, 774-781.

Hollon, S. D., \& Kriss, M. R. (1984). Cognitive factors in clinical research and practice. Clinical Psychology Review, 4, 35-76.

Hollon, S. D., Stewart, M. O., \& Strunk, D. (2006). Enduring effects for cognitive behavior therapy in the treatment of depression and anxiety. Annual Review of Psychology, 57, 285-315.

Imber, S. D., Pilkonis, P. A., Sotsky, S. M., Elkin, I., Watkins, J. T., Collins, J. F., et al. (1990). Mode-specific effects among three treatments for depression. Journal of Consulting and Clinical Psychology, 58, 352-359.

Ingram, R. E., \& Hollon, S. D. (1986). Cognitive therapy for depression from an information processing perspective. In R. E. Ingram (Ed.), Information processing approaches to clinical psychology (pp. 259281). San Diego, CA: Academic Press.

Jacobson, N. S., Dobson, K. S., Truax, P. A., Addis, M. E., Koerner, K., Gollan, J. K., et al. (1996). A component analysis of cognitivebehavioral treatment for depression. Journal of Consulting and Clinical Psychology, 64, 295-304.

Keller, M. B., Lavori, P. W., Friedman, B., Nielsen, E., Endicott, J., McDonald-Scott, P., \& Andreasen, N. C. (1987). The longitudinal interval follow-up evaluation: A comprehensive method for assessing outcome in prospective longitudinal studies. Archives of General Psychiatry, 44, 540-548.

Keller, M. B., Lavori, P. W., Rice, J., Coryell, W., \& Hirschfeld, R. M. (1986). The persistent risk of chronicity in recurrent episodes of nonbipolar major depressive disorder: A prospective follow-up. American Journal of Psychiatry, 143, 24-28.

Kessler, R. C., Berglund, P., Demler, O., Jin, R., Merikangas, K. R., \& Walters, E. E. (2005). Lifetime prevalence and age-of-onset distributions of $D S M-I V$ disorders in the National Comorbidity Survey Replication. Archives of General Psychology, 62, 593-602.

Kessler, R. C., Chiu, W. T., Demler, O., Merikangas, K. R., \& Walters, E. E. (2005). Prevalence, severity, and comorbidity of 12-month $D S M-I V$ disorders in the National Comorbidity Survey Replication. Archives of General Psychology, 62, 617-627.

Kraemer, H. C., Stice, E., Kazdin, A., Offord, D., \& Kupfer, D. (2001). How do risk factors work together? Mediators, moderators, and independent, overlapping, and proxy risk factors. American Journal of Psychiatry, 158, 848-856.

LeDoux, J. (1996). The emotional brain: The mysterious underpinnings of emotional life. New York: Simon \& Schuster.

Linehan, M. M. (1993). Cognitive-behavioral treatment of borderline personality disorder. New York: Guilford.

Moore, R. G., Hayhurst, H., \& Teasdale, J. D. (1996). Measure of Awareness and Coping in Autobiographical Memory: Instructions for administering and coding. Unpublished manuscript, Department of Psychiatry, University of Cambridge, Cambridge, England.

Petersen, T., Feldman, G., Harley, R., Fresco, D. M., Graves, L., Holmes, L., et al. (2007). Extreme response style in recurrent and/or chronically depressed patients-Change with acute antidepressant treatment and stability during continuation treatment. Journal of Consulting and Clinical Psychology, 75, 145-153.

Peterson, C., Semmel, A., von Baeyer, C., Abramson, L. Y., Metalsky, G. I., \& Seligman, M. E. P. (1982). The Attributional Style Questionnaire. Cognitive Therapy and Research, 6, 287-300.

Prokofiev, S. (1987). Russia under the Mongolian yoke [Alexander Nevsky]. Cleveland, OH: Telarc. (Original work published 1934)

Rottenberg, J., Gross, J. J., \& Gotlib, I. H. (2005). Emotion context insensitivity in major depressive disorder. Journal of Abnormal Psychology, 114, 627-639.

Rottenberg, J., Kasch, K. L., Gross, J. J., \& Gotlib, I. H. (2002). Sadness and amusement reactivity differentially predict concurrent and prospective functioning in major depressive disorder. Emotion, 2, 135-146.
Safran, J. D., \& Segal, Z. V. (1990). Interpersonal process in cognitive therapy. New York: Basic Books.

Safran, J. D., Vallis, T. M., Segal, Z. V., \& Shaw, B. F. (1986). Assessment of core cognitive processes in cognitive therapy. Cognitive Therapy and Research, 10, 509-526.

Samoilov, A., \& Goldfried, M. R. (2000). Role of emotion in cognitivebehavior therapy. Clinical Psychology: Science and Practice, 7, 373-385.

Segal, Z. V., Gemar, M., \& Williams, S. (1999). Differential cognitive response to a mood challenge following successful cognitive therapy or pharmacotherapy for unipolar depression. Journal of Abnormal Psychology, 108, 3-10.

Segal, Z. V., Kennedy, S., Gemar, M., Hood, K., Pedersen, R., \& Buis, T. (2006). Cognitive reactivity to sad mood provocation and the prediction of depressive relapse. Archives of General Psychiatry, 63, 749-755.

Segal, Z. V., Williams, J. M. G., \& Teasdale, J. D. (2002). Mindfulnessbased cognitive therapy for depression: A new approach to preventing relapse. New York: Guilford Press.

Simons, A. D., Garfield, S. L., \& Murphy, G. E. (1984). The process of change in cognitive therapy and pharmacotherapy for depression: Changes in mood and cognition. Archives of General Psychiatry, 41, $45-51$.

Solomon, D. A., Keller, M. B., Leon, A. C., Mueller, T. I., Lavori, P. W., Shea, M. T., et al. (2000). Multiple recurrences of major depressive disorder. American Journal of Psychiatry, 157, 229-233.

Teasdale, J. D. (1999). Emotional processing, three modes of mind, and the prevention of relapse in depression. Behaviour Research and Therapy, 37, S53-S78.

Teasdale, J. D., Moore, R. G., Hayhurst, H., Pope, M., Williams, S., \& Segal, Z. V. (2002). Metacognitive awareness and prevention of relapse in depression: Empirical evidence. Journal of Consulting and Clinical Psychology, 70, 275-287.

Teasdale, J. D., Scott, J., Moore, R. G., Hayhurst, H., Pope, M., \& Paykel, E. S. (2001). How does cognitive therapy prevent relapse in residual depression? Evidence from a controlled trial. Journal of Consulting and Clinical Psychology, 69, 347-357.

Vallis, T. M., Shaw, B. F., \& Dobson, K. S. (1986). The Cognitive Therapy Scale: Psychometric properties. Journal of Consulting and Clinical Psychology, 54, 381-385.

Ventura, J., Liberman, R. P., Green, M. F., Shaner, A., \& Mintz, J. (1998). Training and quality assurance with the Structured Clinical Interview for DSM-IV (SCID-I/P). Psychiatry Research, 79, 163-173.

Weissman, A. N. (1979). The Dysfunctional Attitude Scale: A validation study. Dissertation Abstracts International, 40, 1389-1390.

Weissman, A. N., \& Beck, A. T. (1978, March). Development and validation of the Dysfunctional Attitude Scale: A preliminary investigation. Paper presented at the meeting of the American Educational Research Association, Toronto, Ontario, Canada.

Wells, A. (1990). Panic disorder in association with relaxation-induced anxiety: An attentional training approach to treatment. Behavior Therapy, 21, 273-280.

Wells, A., \& Matthews, G. (1994). Attention and emotion: A clinical perspective. Hove, England: Erlbaum.

Westermann, R., Spies, K., Stahl, G., \& Hesse, F. W. (1996). Relative effectiveness and validity of mood induction procedures: A metaanalysis. European Journal of Social Psychology, 26, 557-580.

Zimmerman, M., Chelminski, I., \& Posternak, M. (2004). A review of studies of the Hamilton Depression Rating Scale in healthy controls: Implications for the definition of remission in treatment studies of depression. Journal of Nervous and Mental Disease, 192, 595-601.

Received August 18, 2006

Revision received February 2, 2007

Accepted February 12, 2007 